\title{
Découverte d'une somptueuse maison tardo-républicaine à
}

\section{Ostie}

In: Comptes-rendus des séances de l'Académie des Inscriptions et Belles-Lettres, 147e année, N. 2, 2003. pp. 695713.

Citer ce document / Cite this document :

Morard Thomas, Wavelet David. Découverte d'une somptueuse maison tardo-républicaine à Ostie. In: Comptes-rendus des séances de l'Académie des Inscriptions et Belles-Lettres, 147e année, N. 2, 2003. pp. 695-713.

doi : $10.3406 /$ crai.2003.22594

http://www.persee.fr/web/revues/home/prescript/article/crai 0065-0536 2003 num_147_2 22594 


\title{
COMMUNICATION
}

\author{
DÉCOUVERTE D'UNE SOMPTUEUSE MAISON TARDO-RÉPUBLICAINE \\ A OSTIE, \\ PAR M. THOMAS MORARD*
}

Après plusieurs saisons d'activité, la mission archéologique de l'Université de Lyon II, dirigée par le Professeur Jean-Marc Moret, est en mesure de présenter les premiers résultats et les perspectives de développement de son projet de recherche engagé sur le site de la Schola du Trajan (IV, 5, 15-17) à Ostie'. L'objectif de ce projet de recherche, déterminé et poursuivi en étroite collaboration avec la Surintendance archéologique d'Ostie ${ }^{2}$, prévoit l'étude systématique d'une parcelle urbaine particulière, située le long du Decumanus Maximus, dans le quartier stratégiquement sensible de la Porta Marina. Le site sélectionné, partiellement fouillé durant l'hiver 1938-1939, conserve en effet les restes d'au moins trois édifices successifs : la Schola du Trajan (II ${ }^{\mathrm{e}}$-IV $\mathrm{e}$ s. ap. J.-C.), édifice public monumental, vraisemblablement corporatif, la Domus à péristyle ( $\mathrm{I}^{\mathrm{er}}-\mathrm{II}^{\mathrm{e}} \mathrm{s}$. ap. J.-C.) et la Domus aux bucranes ( $\mathrm{I}^{\text {er }}$ s. av. J.-C.), témoins essentiels de l'architecture domestique de la colonie romaine ${ }^{3}$. Compte tenu de l'ampleur du

\footnotetext{
- Pour des raisons indépendantes de sa volonté, David Wavelet n'a pas été à même de mettre la dernière main à la communication qu'il avait présentée à l'Académie le 2 mai 2003. Thomas Morard lui a fait l'amitié de ré-élaborer le texte pour l'impression. Je l'en remercie au nom de la mission. (Jean-Marc Moret).

1. Pour en savoir plus sur ce projet de recherche, consulter en premier lieu : Th. Morard et D. Wavelet, «Un nouveau projet archéologique de l'Université de Lyon II », MEFRA 113.1 (2001), p. 477-481; Th. Morard et D. Wavelet, «Prolégomènes à l'étude de la Schola du Trajan à Ostie ", MEFRA 114.2 (2002), p. 759-815; Th. Morard, "Ostie : la reprise des fouilles sur le site de la Schola du Trajan (Reg. IV, IS. V, 15-17) ", MEFRA 115.1 (2003), p. 433-443.

2. Nous réitérons, à cette occasion, notre vive gratitude à A. G. Zevi, A. Pellegrino et S. Falzone, ainsi qu'à tous les collaborateurs de la Surintendance d'Ostie, engagés d'une manière ou d'une autre dans notre entreprise. Une pensée toute particulière s'adresse à E. J. Shepherd et P. Olivanti, sans les conseils avisés desquelles l'indispensable étude des archives n'aurait tout simplement pas été possible.

3. La nomenclature de ces trois édifices est bien évidemment moderne. La Schola du Trajan (IV, 5, 15) fut ainsi désignée en raison de la découverte d'une statue cuirassée de
} 
dossier et de la nature des découvertes les plus récentes (fig. 1), le présent rapport se concentrera sur les premières phases d'occupation de la parcelle urbaine de la Schola du Trajan, à savoir la construction de la Domus aux bucranes, son aménagement et sa destruction, provoquée selon toute vraisemblance par la volonté de rehausser le niveau de sol de ce quartier d'Ostie et la mise en chantier de la Domus à péristyle.

La découverte de l'ensemble domestique tardo-républicain de la Domus aux bucranes représente sans aucun doute une étape essentielle dans la connaissance d'Ostie au temps de P. Lucilius Gamala et de M. Tullius Cicero. De plus, la variété et la qualité exceptionnelle de l'appareil décoratif de cette maison particulière s'imposent déjà comme des arguments majeurs au dossier de l'étude des pavements, des enduits peints et des stucs de deuxième style.

\section{Contexte de découverte de la Domus aux bucranes}

La présence de structures tardo-républicaines, sur le site de la Schola du Trajan, avait déjà été mentionnée dans le rapport d'activité rédigé durant l'hiver 1938-1939, au cours des fouilles menées alors à cet endroit ${ }^{4}$. En date du 3 novembre 1938, G. Becatti y rapporte en effet la découverte de structures plus anciennes que celles de la Domus à péristyle :

« Nel piazzale dietro l'edificio a nicchie [la Schola du Trajan] sul lato $S$ del Decumanus nei saggi che si stanno praticando per ricercare i resti della casa precedente più antica, in una fossa praticata proprio davanti all'ingresso $S$ dell'edificio è venuto in luce un resto di muro ancora più antico della casa con peristilio e stanze a mosaico [ $l a$ Domus à péristyle], con la faccia $\mathrm{S}$ rivestita di fine intonaco dipinto a colori vivi con zoccolo imitante il rivestimento di giallo antico, e sopra riquadri rossi e lesure di verde. In questo saggio si è trovato un frammento di lucerna bilicne a vernice nera con decorazione in rilievo che si data alla fine della Repubblica e va in relazione probabilmente con la parete dipinta ».

\footnotetext{
l'empereur durant les fouilles d'octobre 1938. A la même période, à un niveau inférieur, furent mis au jour les restes d'un édifice plus ancien, qui fut dénommé Maison à péristyle. La Domus aux bucranes (IV, 5, 17 bis), découverte en 1998, tire son nom de la frise de bucranes et de patères, l'un des plus séduisants décors peints de cette maison tardo-républicaine.

4. Pour un compte rendu précis de l'historique des fouilles de 1938-1939, voir Th. Morard et D. Wavelet, « Prolégomènes », loc. cit. (n. 1), p. 787-807, not. 805-806.
} 


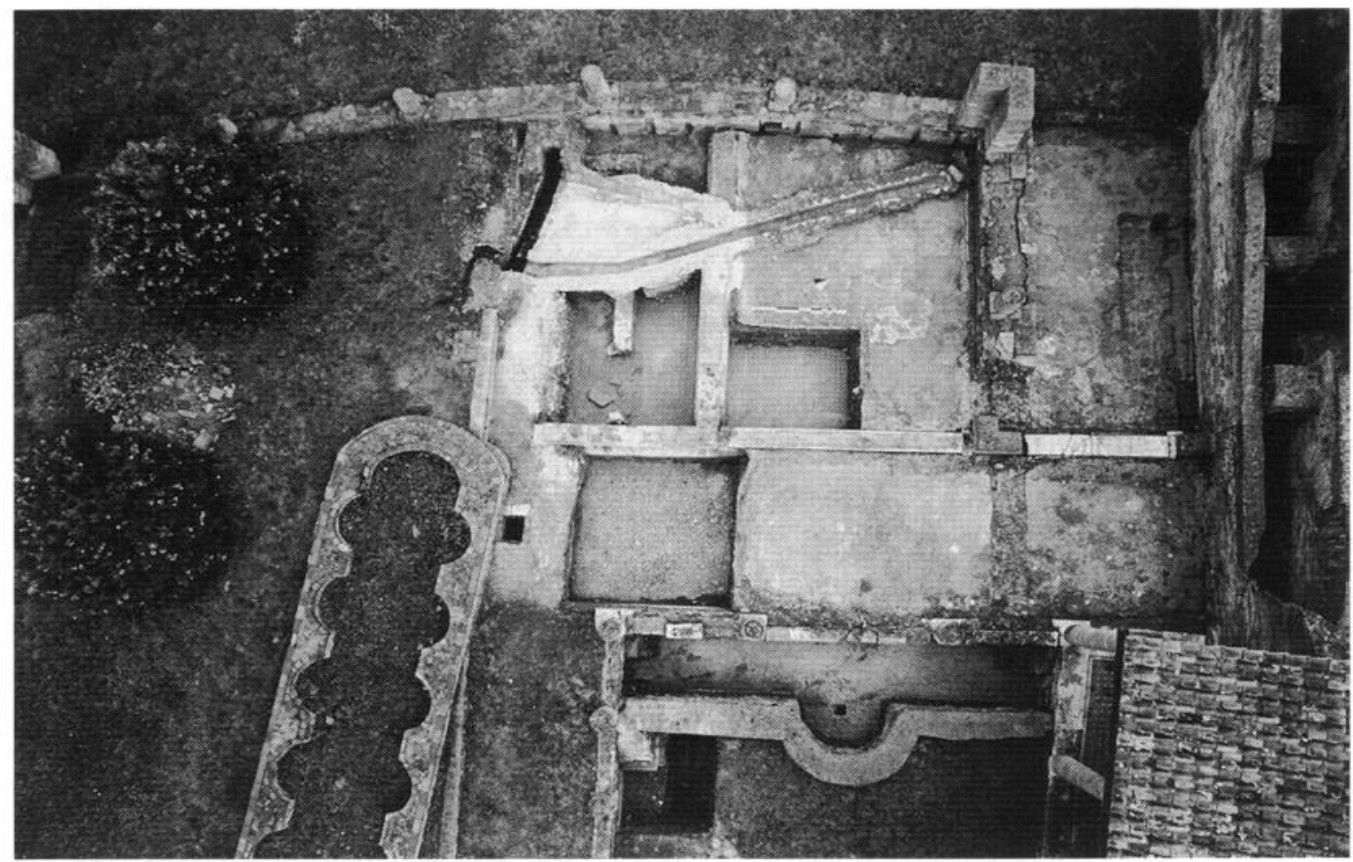

FIG. 1. - Le chantier de fouilles après la campagne TRA02 : les trois niveaux d'occupation (Schola du Trajan, Domus à péristyle et Domus aux bucranes) sont désormais bien définis. Prise de vue effectuée au moyen d'un ballon atmosphérique.

Le 9 novembre 1938, il est fait état de la mise au jour d'un puits tardo-républicain intégré, dans un second temps, au complexe de la Domus à péristyle :

« Nel piazzale dietro l'edificio a nicchie [la Schola du Trajan] continuano i saggi per ricercare i resti delle costruzioni più antiche e nell'angolo SE della fascia di travertino su cui poggiavano le colonne del peristilio della seconda casa [la Domus à péristyle] è venuto in luce ad un livello più basso un pozzo circolare in reticolato che era stato rispettato dalle fondazioni del peristilio della 2a casa, che qui formavano una volticella a botte impostata pure su muri in reticolato. Sull'orlo del pozzo si sono trovati dei vasetti fittili poggiati e anneriti. Due coppe su alto piede, con corpo ad imbuto e labbro rientrante, di cui una con decorazione esterna sulle spalle a linguette irregolari in rilievo. Una coppa bassa verniciata di rosso chiaro con decorazione di segmenti incisi sul bordo. Due lucernette fittili del tipo con il c.s. candelabro a sette bracci ».

Une série de photographies d'archives, prises à la même époque, permettent de confirmer qu'en plusieurs endroits les investigations avaient alors été menées en-dessous du niveau de 


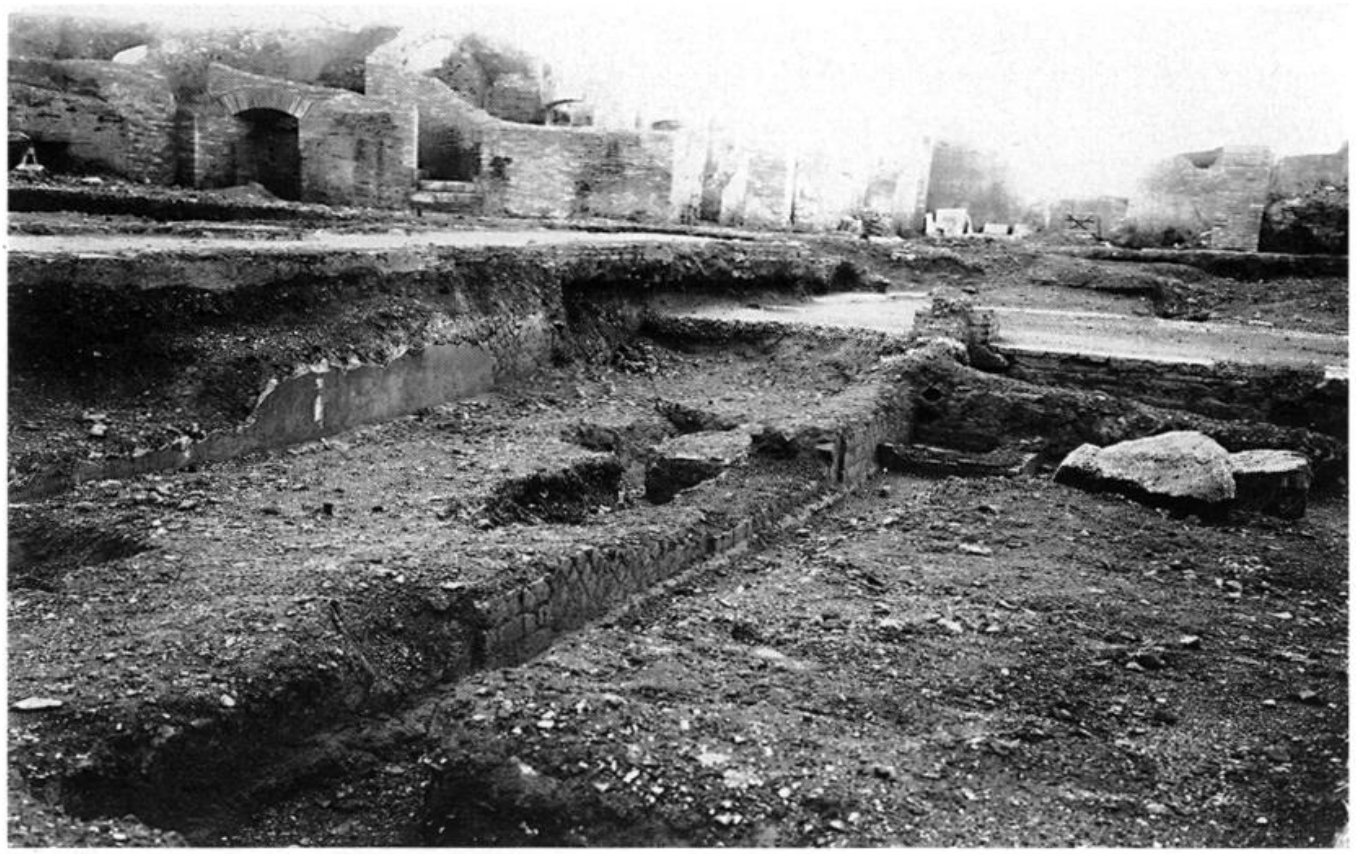

Fig. 2. - Cliché d'archives SBAO B2794. L'angle occidental du péristyle et de l'hortus de la Domus à péristyle.

sol de la Domus à péristyle. Le cliché photographique SBAO B2794 (fig. 2) laisse clairement apparaître, sous le portique de la Domus à péristyle, en contrebas de l'euripe de la Schola du Trajan, un mur en opus quasi reticulatum, qu'il faut selon toute vraisemblance rattacher au péristyle de la Domus aux bucranes. Il conserve sur presque toute la surface excavée sa décoration pariétale, taillée au niveau du pavement de la Domus à péristyle.

Aussi étonnant que cela puisse paraître, ces observations n'ont jamais été exploitées ni publiées ; elles sont ainsi tombées dans l'oubli. L'ouverture récente d'un sondage stratigraphique dans le portique de la Domus à péristyle a permis de reprendre le dossier dans son ensembles. La découverte d'un pavement en opus signinum à tesselles et éclats, à quelque $150 \mathrm{~cm}$ en-dessous du niveau de sol de la Domus à péristyle, rappelait de manière définitive que la parcelle urbaine de la Schola du Trajan était déjà

5. Ces fouilles ont été réalisées par une équipe internationale, sous la responsabilité scientifique du Professeur Clemens Krause. Voir L. Chrzanovski, C. Krause, A. Pellegrino, "Nuove indagini nella Schola del Traiano ad Ostia », dans R. F. Docter et E. M. Moormann (éd.). Proceedings of the $X V^{\text {th }}$ International Congress of Classical Archaeology, Amsterdam. 
occupée à l'époque républicaine ${ }^{6}$. L'hypothèse qui rattachait les nombreux fragments d'enduits peints et de stucs, retrouvés dans le remblai que scellait ce pavement, au décor de l'édifice tardorépublicain redécouvert, permettait d'envisager qu'il s'agissait là d'une demeure particulièrement somptueuse, dont l'appareil décoratif de deuxième style apparaissait remarquablement bien conservé.

La première campagne de fouilles, spécifiquement consacrée aux ruines de la Domus aux bucranes, ne fut organisée que durant l'été 2002 , dans le cadre du projet de recherche mis en œuvre par le Professeur Jean-Marc Moret pour ses étudiants lyonnais ${ }^{7}$. Les quatre sondages alors entrepris en-dessous du niveau de sol de la Domus à péristyle ont révélé la séquence stratigraphique suivante :

1. pavements (ou couche végétale de l'hortus) (environ 230$240 \mathrm{~cm} \mathrm{slm}$ ) et préparations de pavement de la Domus $\dot{a}$ péristyle;

2. couche de remblais, épaisse de près de $150 \mathrm{~cm}$, composée d'une terre jaunâtre, très argileuse et compacte, alternant avec des poches de terre brune et sablonneuse, truffées de matériel archéologique, formant un ensemble cohérent. essentiellement composé d'éléments tardo-républicains (céramiques, enduits peints et stucs, monnaies, petits objets et éléments de construction);

3. pavements (ou couche végétale de l'hortus) (environ $80-90 \mathrm{~cm}$ slm) et élévations (environ $150-160 \mathrm{~cm}$ slm) de la Domus aux bucranes, qui conservaient in situ leurs revêtements d'enduits peints.

July 12-17, 1998. Classical Archaeology towards the Third Millenium : Reflections and Perspectives, Amsterdam 1999, p. 117-118 ; L. Chrzanovski, C. Krause, A. Pellegrino, "Les nouvelles fouilles de la Schola del Traiano : premiers résultats", dans Ostia, Port et Porte de la Rome antique, Genève, 2001, p. 74-78 et catalogue II, p. 395-396; M. David, « La successione dei livelli pavimentali nel perimetro della Schola del Traiano ", MNIR 58, p. 66-70.

6. Cette réalité permet de rattacher les murs en opus incertum, depuis longtemps documentés dans l'espace souterrain 19, à la Domus aux bucranes.

7. La mission archéologique de l'Université de Lyon II s'est attachée, tout d'abord, à réaliser une synthèse des fouilles qui avaient été entreprises sur le site de la Schola du Trajan, notamment par l'étude des archives de la Surintendance, puis elle a tenté de combler les importantes lacunes constatées dans la documentation idoine, notamment dans le catalogue du mobilier archéologique. C'est alors, et alors seulement, que la reprise du chantier de fouilles a été envisagée (étude et mise en évidence des structures de la Schola du Trajan, de la Domus à péristyle et de la Domus aux bucranes). 
De toute évidence, les élévations en opus incertum de la Domus aux bucranes avaient été arasées à une hauteur d'environ $80 \mathrm{~cm}$ lors du chantier de la Domus à péristyle. Tous les éléments de construction qui ne pouvaient alors être récupérés ont été abandonnés à même le sol ou intégrés à la couche de remblais qui devait en recouvrir les ruines. Cette couche particulière fut ensuite scellée sous les pavements de la Domus à péristyle.

\section{Description des structures découvertes de la Domus aux bucranes}

A ce stade de l'enquête, les ruines des élévations, les seuils et les niveaux de sol de diverses natures ont d'ores et déjà permis de distinguer cinq espaces particuliers de la Domus aux bucranes (fig. 3). Dans la partie méridionale de l'édifice tardo-républicain, un large péristyle (sondages $\mathrm{G}$ et $\mathbf{H}$ ) enfermait un hortus (sondage $\mathrm{H}$ ) agrémenté de différentes structures horticoles. Vers le corps du bâtiment, ce péristyle communiquait directement avec une grande pièce axiale (sondages $E$ et $F$ ), probablement le tablinum de la Domus aux bucranes. De plus, deux petites pièces latérales (sondages $\mathrm{D}$ et $\mathrm{F}$ ), qu'il faut selon toute vraisemblance interpréter comme des cubicula, s'ouvraient sur le flanc occidental de ce tablinum. Le plan de la Domus aux bucranes devait correspondre au plan traditionnel de la domus tardo-républicaine, décrite théoriquement par Vitruve, dont la cité de Pompéi conserve de nombreuses variantes.

Une récente chronique des $M E F R A^{8}$ analyse, sondage par sondage, l'ensemble des structures de la Domus aux bucranes mises au jour au cours de ces dernières années. Nous proposons ici de reconsidérer le dossier d'un point de vue différent, en mettant en évidence les éléments les plus significatifs de l'appareil décoratif de l'édifice tardo-républicain : les pavements (opus signinum et opus tessellatum), ainsi que les ensembles d'enduits peints et de stucs (revêtements de parois et de colonnes, plafond). Il n'est bien évidemment pas encore question de présenter ce matériel de manière exhaustive, ni de livrer l'ensemble des parallèles pertinents aux unités déterminées. L'atelier de restauration 


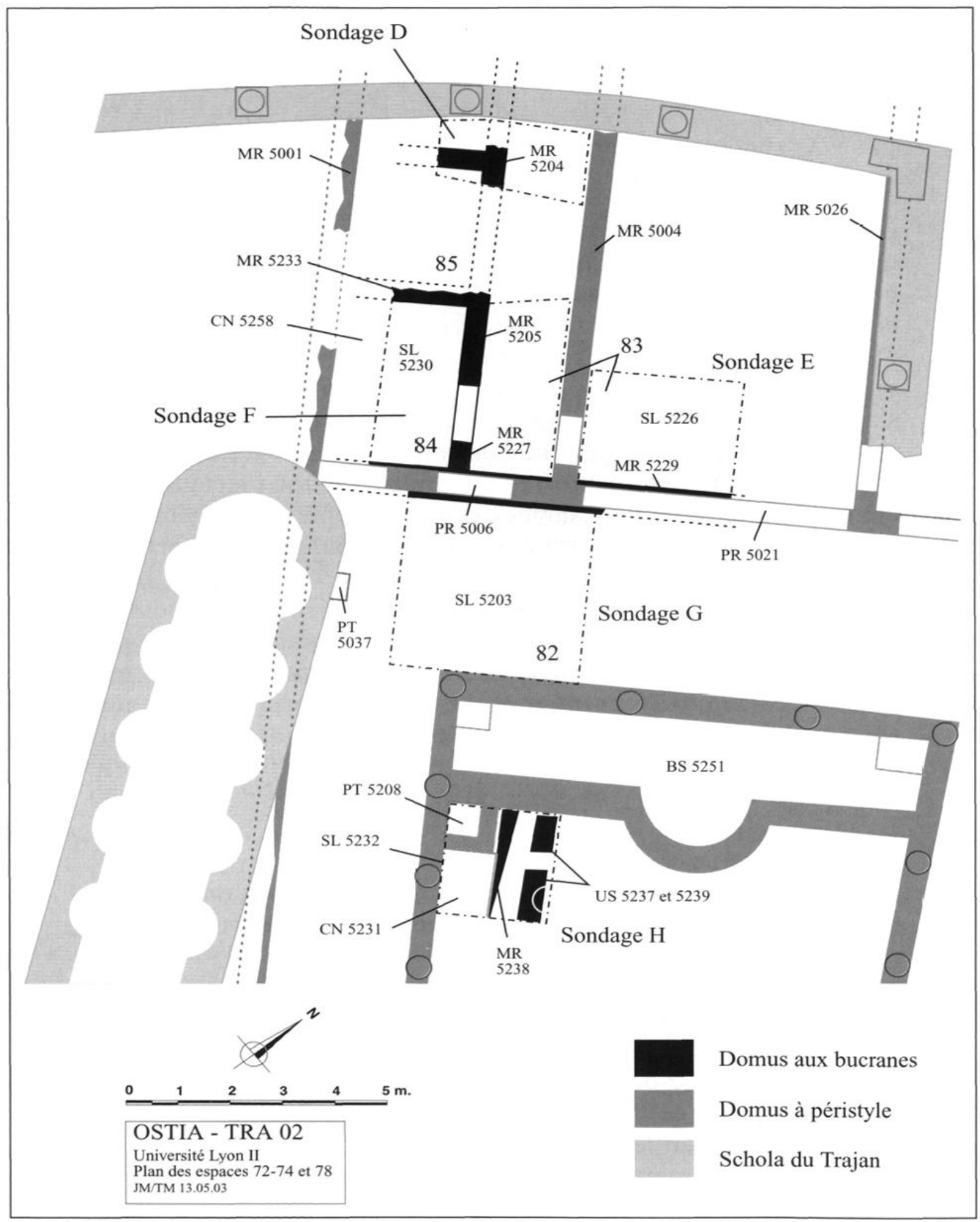

FIG. 3. - Plan des espaces $72,73,74$ et 78 avec indication de l'emplacement des sondages E, F, G et $\mathrm{H}$, ainsi que des nouvelles structures de la Domus aux bucranes. 
doit en effet encore produire l'essentiel de son effort et la recension systématique des complexes tardo-républicains est en cours d'élaboration.

\section{LES PAVEMENTS ET LA COUCHE VÉGÉTALE DE L'HORTUS}

Un pavement en opus signinum à tesselles et éclats ornait le péristyle de la Domus aux bucranes. Le tapis interne, délimité par une ligne de tesselles blanches, présente une composition hétérogène de tesselles et d'éclats de calcaire de différentes natures. Il est intéressant de constater que le pavement du péristyle de la Domus aux bucranes se trouvait en relation directe avec l'hortus qu'il contenait. La couche végétale de cet hortus venait en effet s'appuyer directement contre la bordure convexe de l'opus signinum du péristyle. La fouille de cette couche végétale a révélé la présence d'une olla perforata et d'un amalgame d'éclats de tuf contenant une poche de sable. Ces deux éléments doivent bien évidemment être mis en rapport avec l'aménagement horticole de cet espace.

Le pavement de la grande pièce axiale, probablement le tablinum de la Domus aux bucranes, était composé d'un opus signinum à semis orthogonal de croisettes bichromes (une tesselle centrale noire pour quatre tesselles périphériques blanches) (fig. 4). Deux particularités caractérisent cet individu : l'alternance de tesselles noires et blanches sur la ligne d'encadrement du tapis central et la présence de demi-croisettes (une tesselle centrale noire pour trois tesselles périphériques blanches) en bordure du tapis central.

L'installation d'un important collecteur d'eau lors du chantier de la Domus à péristyle a presque complètement détruit le pavement du premier cubiculum de la Domus aux bucranes. Cet espace particulier, ouvert sur le flanc occidental du tablinum, était jadis composé d'un opus tessellatum bichrome (noir et blanc). Des fragments du tapis de tesselles de cette mosaïque tardorépublicaine, l'une des plus anciennes jamais découvertes sur le site d'Ostie, ont été retrouvés in situ le long des parois de cet espace ou mêlés au remblai qui recouvrait les ruines de la Domus aux bucranes. Ils permettent d'en recomposer assez précisément l'apparence : sur un fond noir se distingue un grand tapis délimité par une bordure blanche et décoré de médaillons géométriques, construits sur les effets d'inversion chromatique de l'encastre- 


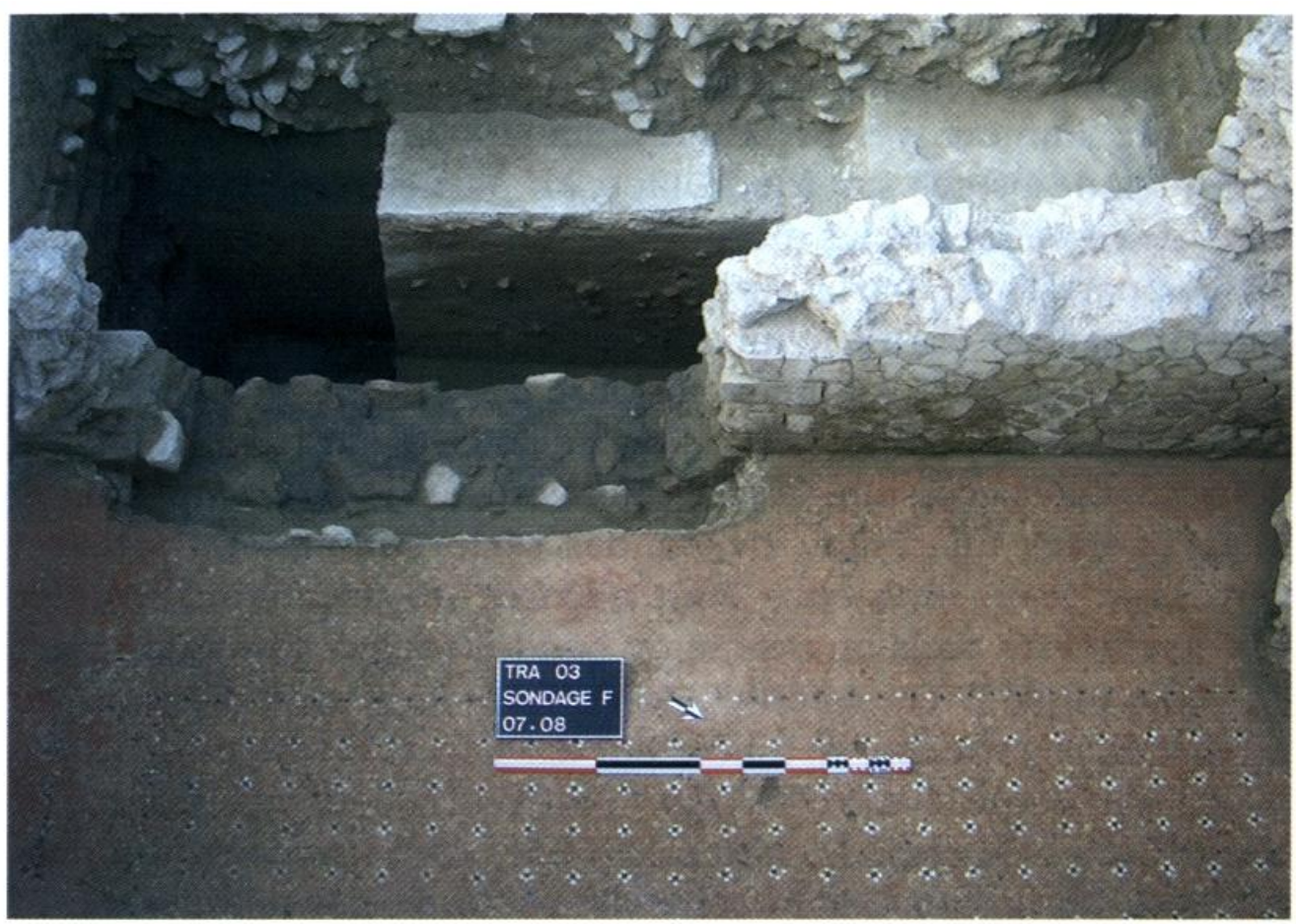

FIG. 4. - Au premier plan : le pavement en opus signinum à semis de croisettes du tablinum de la Domus aux bucranes. Au second plan : le sondage stratigraphique entrepris sous la préparation de pavement de la Domus aux bucranes.

ment de trois carrés alternativement sur le côté et sur la pointe, tantôt noir, tantôt blanc, au centre desquels s'inscrit une fleur blanche à quatre pétales allongés. La spoliation du seuil de la porte ouverte entre le tablinum et le cubiculum mentionnés ne permet malheureusement pas d'en déterminer la nature.

\section{LES ENSEMBLES D'ENDUITS PEINTS ET DE STUCS}

La partie inférieure du décor pariétal du péristyle reste pour l'instant méconnue. La photographie d'archives SBAO B2794 (fig. 2) atteste toutefois que le revêtement d'enduits peints y est conservé in situ sur une hauteur d'environ $120 \mathrm{~cm}$. Une prochaine campagne de fouilles devrait révéler l'aspect de ce bas de paroi, probablement une plinthe sombre et un socle décoré à l'imitation d'un marbre précieux. Les parties médiane et supérieure de cette composition sont en revanche connues, grâce à l'étude systématique des nombreux fragments d'enduits peints jadis arrachés aux parois de ce péristyle et abandonnés dans le remblai qui en 
recouvrait les ruines. Au premier plan, une colonnade d'ordre dorico-toscan soutenait une lourde architrave moulurée. Les effets de lumière y sont admirablement bien rendus par des dégradés de beiges sur fond blanc, en particulier sur les cannelures des colonnes et les moulurations des chapiteaux. Au second plan apparaît une paroi complexe, divisée aux deux tiers de sa hauteur par une corniche moulurée, également rendue en tons de beige et de blanc. La zone supérieure de cette paroi est occupée par une frise de palmettes bordeaux disposées sur fond violet, qui domine une succession de carreaux verts et de boutisses rouges, intégrés dans un réseau de bandes réciproquement rouges et vertes; la zone inférieure était décorée de grands orthostates violets intégrés dans un réseau de bandes rouges. Des guirlandes de vigne ou de chêne, accrochées derrière le fût des colonnes, au niveau de la corniche, en animaient la surface. Quelques oiseaux, peut-être des pics-verts, y étaient perchés. Le bossage interne et externe des carreaux, des boutisses et des orthostates est toujours souligné par des jeux de lumière : les arêtes y sont rendues tantôt en blanc, tantôt en noir. C'est une constante dans l'appareil décoratif de la Domus aux bucranes. En regardant de près le cliché SBAO B2794, des structures verticales claires se distinguent sur un fond sombre. Il s'agit là, selon toute vraisemblance, de la partie basse des fûts cannelés des colonnes dorico-toscanes et des grands orthostates violets. Un autre ensemble d'enduits peints a également été découvert dans le remblai de ce péristyle : une frise dorique décorée de bucranes et de patères, dont la réalisation a impliqué l'usage d'une gamme de couleurs subtile et variée (fig. 5). Certains fragments, marqués d'un angle ouvert à $90^{\circ}$ ou de segments de courbe, permettent de supposer que cette frise décorait un petit édicule, peut-être un lararium. Le fût des colonnes du péristyle de la Domus aux bucranes était composé de briques de terre cuite ordonnées selon une séquence régulière d'une assise épaisse pour deux assises fines. Un revêtement de stuc recouvrait cet appareil particulier : la partie basse de la colonne, lisse et pourpre ; la partie haute, cannelée et blanche. Les chapiteaux étaient encore une fois d'ordre dorico-toscan. L'architrave présentait des moulurations de stuc sur sa face interne et des plaques de terre cuite architecturales sur sa face externe. Mentionnons enfin dans cet espace la découverte de quelques fragments d'un plafond peint sur support de roseaux et décoré d'un entrelacs de lignes circulaires blanches sur un fond violet. 


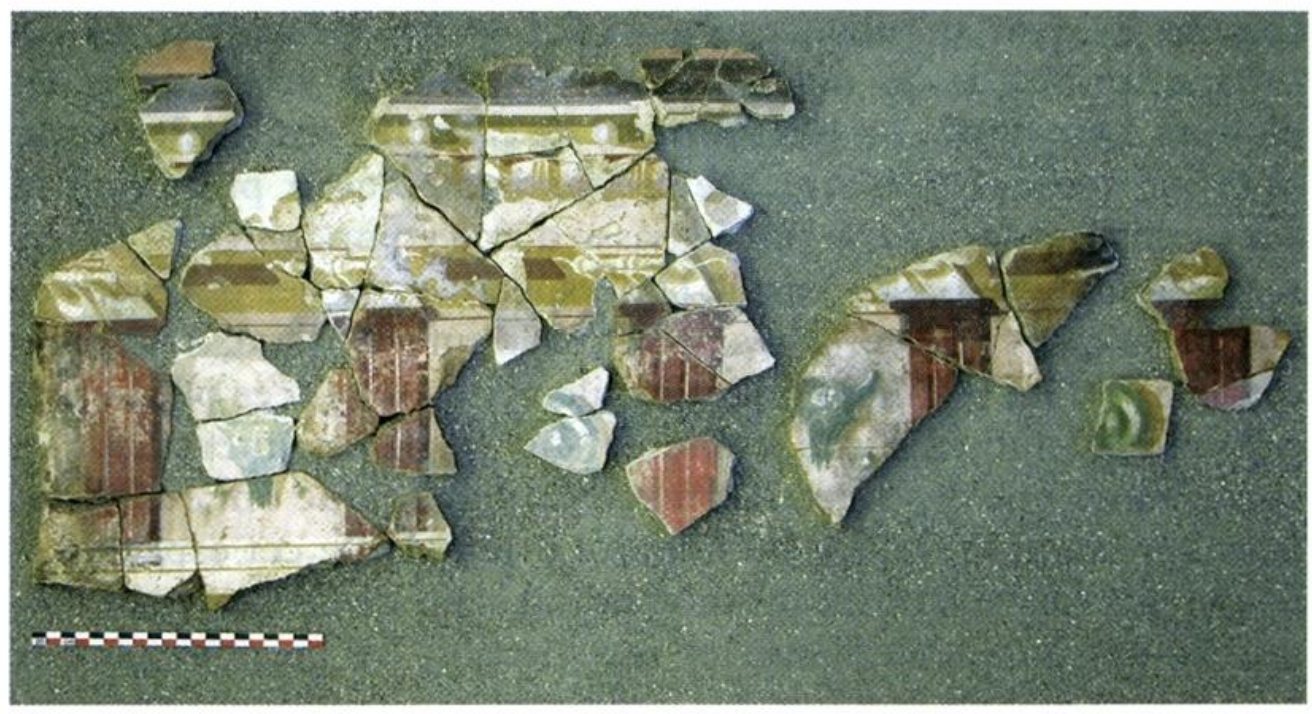

FIG. 5. - Recomposition d'une partie de la frise dorique, dont les métopes roses sont alternativement décorées de bucranes et de patères verts.

On peut s'étonner de constater qu'il s'agit là des seuls éléments de plafond découverts pour l'instant dans les ruines de la Domus aux bucranes.

La décoration des parois du tablinum de la Domus aux bucranes peut être presque entièrement reconstituée en réunissant les éléments livrés par un panneau retrouvé in situ et les nombreux fragments découverts dans le remblai lié à la destruction de l'édifice. Cette fresque tardo-républicaine, d'excellente facture, présentait un socle peint à l'imitation du marbre « giallo antico », souligné par une plinthe noire (fig. 6). Le socle servait de base à une succession de grands orthostates violets et d'étroites lésènes vertes, insérés dans un réseau de bandes rouges. Une corniche moulurée, rendue dans des dégradés de beiges, dominait la composition. La partie haute, dont la reconstitution reste à ce jour hypothétique, devait présenter une succession de carreaux et de boutisses rouges, coiffés d'une corniche de stuc mouluré. Il ne fait désormais aucun doute que la paroi mise au jour durant l'hiver 1938 et décrite dans le rapport d'activité précédemment cité, appartenait à cet espace particulier.

Le décor pariétal du cubiculum fouillé de la Domus aux bucranes devait être particulièrement complexe. Plusieurs ensembles ont été individualisés, mais les liens qui les rapprochent restent pour l'instant incertains. A ce stade de l'enquête, il 
semble évident qu'une césure marquait la décoration de cet espace, au-dessus du niveau du socle, entre le vestibule et les alcôves. Une partie du bas de paroi de cette fresque était encore une fois conservée in situ. Deux grands panneaux présentaient, sur une plinthe rouge, la partie basse d'une composition architecturale rendue selon les lois de la perspective par des dégradés de violet, de gris et de vert. Les moulures d'un piédestal, d'un pilier d'angle et d'un podium étaient habilement soulignées par l'usage des jeux d'ombres et de lumières (fig. 7). Il est intéressant de mentionner que des traces de restauration antique ont été observées sur la plinthe rouge de la paroi orientale de cet espace. Les fragments provenant du remblai permettent de recomposer l'essentiel des zones médianes et supérieures. Le premier plan se caractérise encore une fois par la présence de colonnes cannelées supportant une architrave moulurée, tous deux rendus en dégradé de beige et de blanc. Au second plan, une lourde corniche décorée d'une frise d'oves et de fers de lance divisait horizontalement la paroi. La zone inférieure présentait des orthostates rouge cinabre de dimension relativement modeste, intégrés dans un réseau de bandes blanches. Ces orthostates étaient encadrés par un réseau tout à fait original de carreaux et de boutisses, verts, violets et jaunes. La zone supérieure était composée d'une succession de quatre registres superposés, composés tantôt de carreaux et de boutisses verts et rouges ou rouges et violets, tantôt de seules boutisses vertes, jaunes et violettes, intercalées de boutisses peintes à l'imitation de trois types de marbres précieux. Ces registres étaient séparés les uns des autres par des bandes noires décorées de subtils motifs floraux, rendus avec des dégradés de violet et de vert. La paroi était dominée par un sombre plafond à caissons. Signalons que cette composition présentait également des vases posés sur une corniche ou une architrave, ainsi qu'une grande baie ouverte sur un jardin idéal, dont il n'est pour l'instant pas possible de déterminer l'emplacement. D'autres fragments attestent enfin la présence de grands orthostates jaunes, intégrés dans un réseau de bandes rouges. La qualité et la variété des fragments de stuc moulurés mis au jour lors de la fouille de ce cubiculum permettent de recomposer plusieurs ensembles remarquables : les piédroits et l'entablement d'une porte décorés d'une tresse et de petites palmettes ; une corniche agrémentée d'une frise d'oves et de fers de lance; une corniche animée de trophées d'armes ; une corniche à caissons 


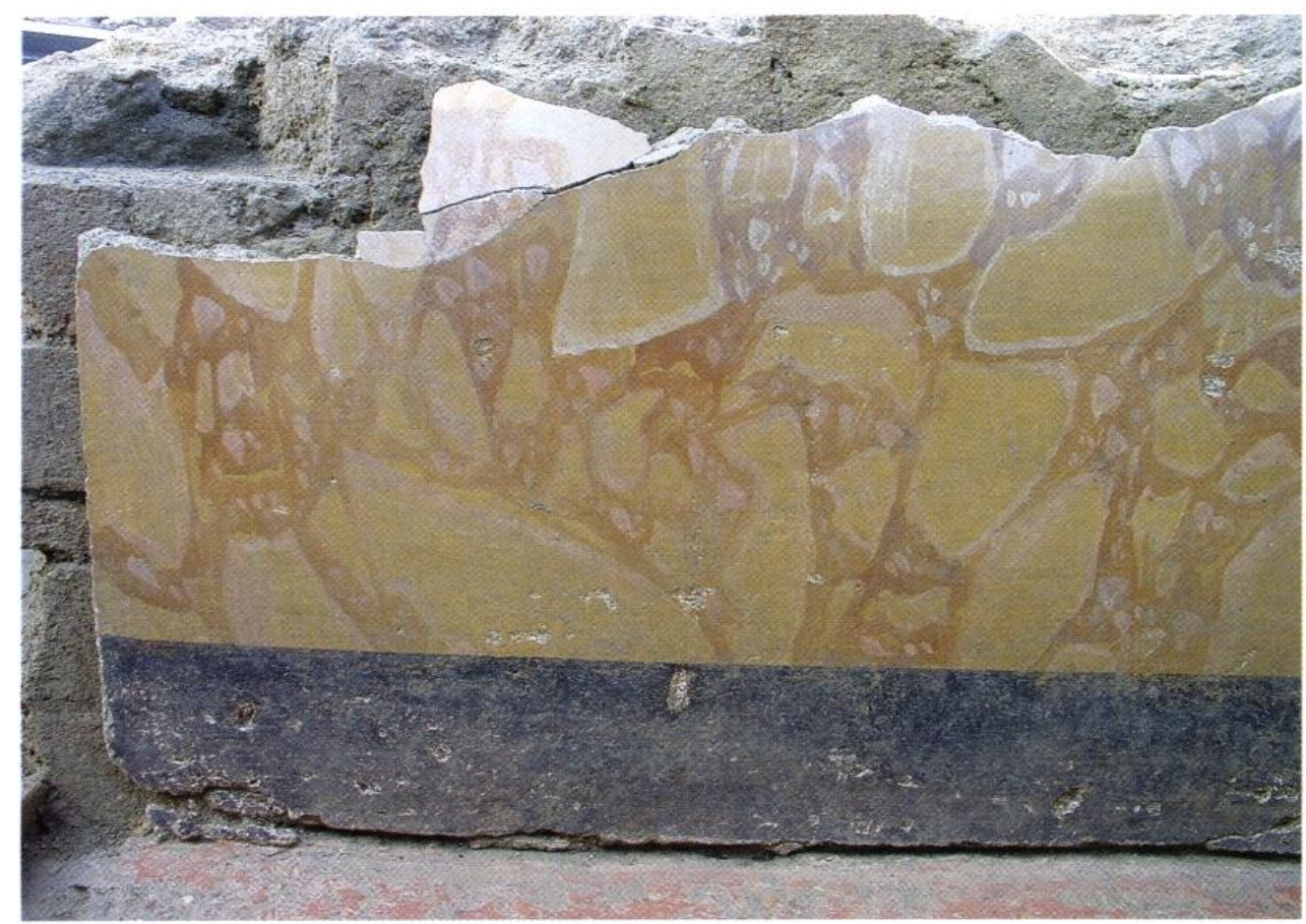

Fig. 6. - Bas de paroi décoré à l'imitation du « giallo antico », conservé in situ sur les ruines de la Domus aux bucranes.

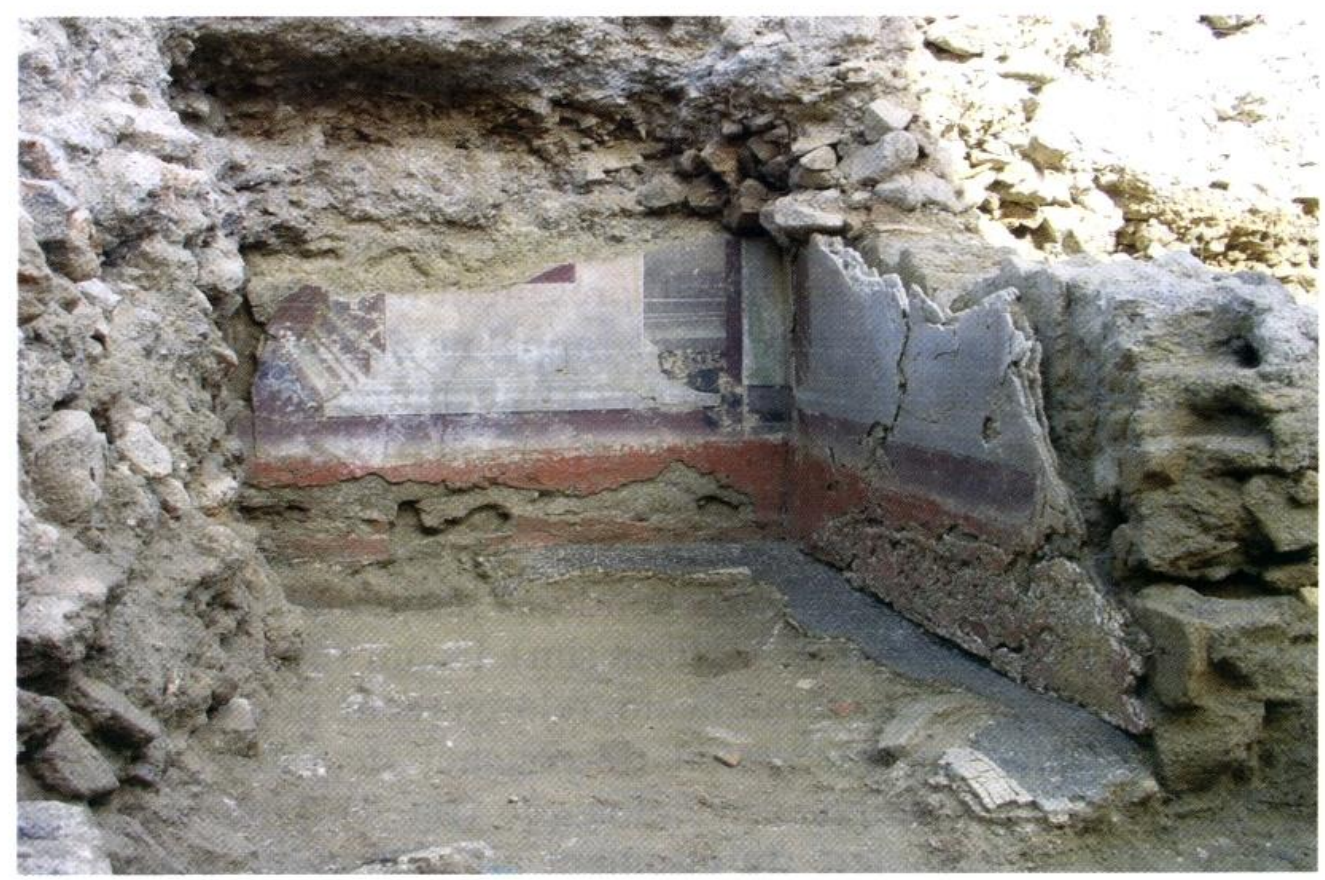

Fig. 7. - Partie inférieure de la décoration pariétale du premier cubiculum de la Domus aux bucranes. La plinthe rouge présente les traces évidentes d'une restauration antique. 


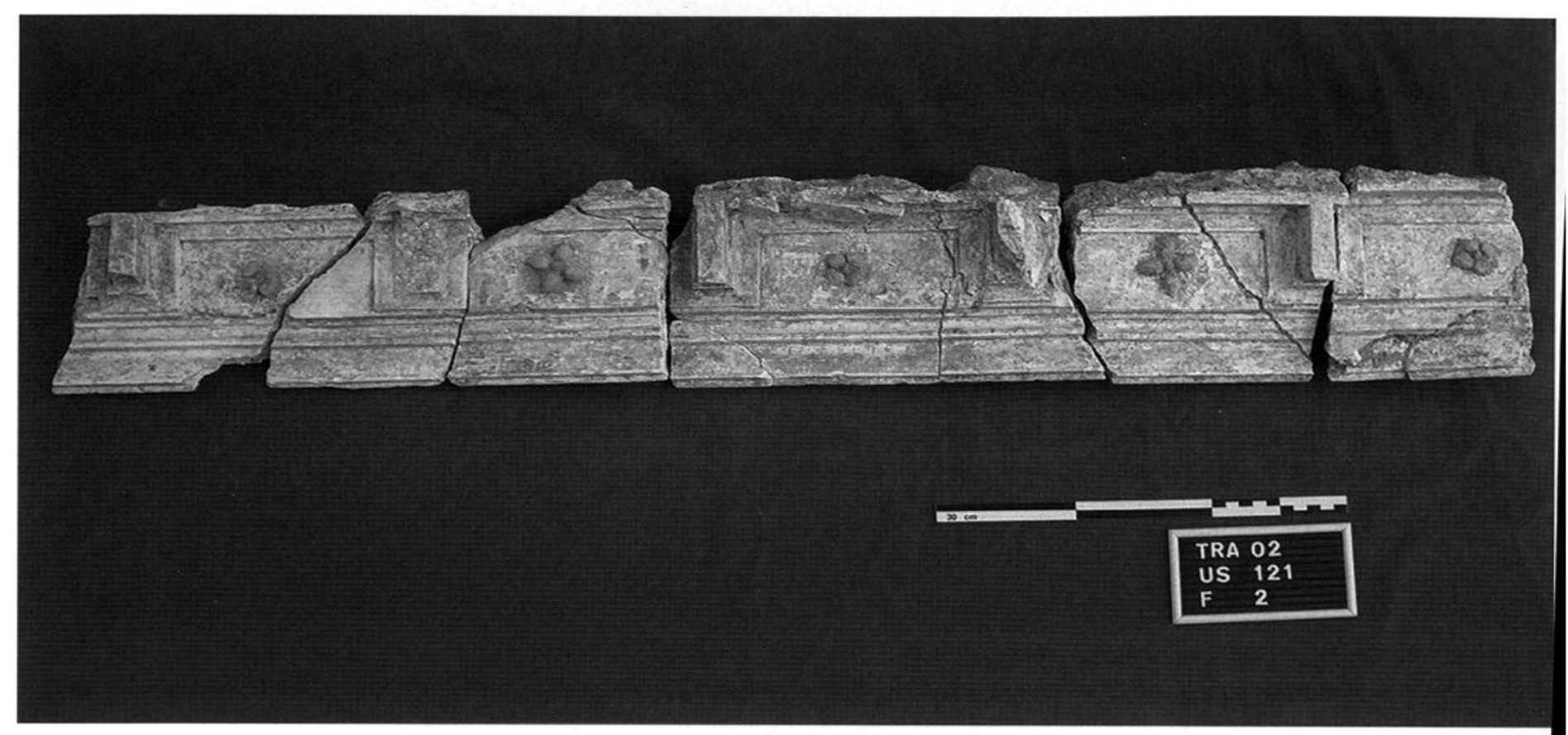

FIG. 8. - Corniche à caissons de stuc décorés alternativement de fleurs à pétales courts et longs. Le cœur de ces éléments végétaux est peint en rouge.

ornés d'une fleur à quatre pétales, tantôt longs, tantôt courts, dont le cour est soigneusement souligné de rouge (fig. 8). Ce dernier ensemble de stuc tardo-républicain est absolument exceptionnel. Il est intéressant de souligner que le pavement de mosaïque de ce cubiculum, précédemment décrit, présentait lui aussi le motif de la fleur à quatre pétales. Cet habile rappel ne doit sans doute rien au hasard. Il n'y a dès lors qu'un pas à franchir pour imaginer, sur le pavement de cet espace, une même alternance de fleurs à pétales courts et à pétales longs.

Les sondages entrepris en-dessous du niveau de sol de la Domus aux bucranes ont été conduits jusqu'au niveau de la nappe phréatique (environ $-50 \mathrm{~cm}$ slm). Ils ont révélé les fondations de l'édifice tardo-républicain, relativement peu profondes, contenues dans une succession de couches de sables plus ou moins riches en mobilier archéologique (fig. 4). Une séquence stratigraphique complète du gisement archéologique a ainsi pu être établie jusqu'au socle géologique, composé d'une couche de sable gris-bleu (environ $-30 / 50 \mathrm{~cm}$ slm), dont la surface était contaminée par quelques éléments anthropiques. 


\section{Constatations préliminaires}

Les bornes chronologiques de la Domus aux bucranes reposent sur un faisceau d'indices complémentaires : références altimétriques, technique de construction des élévations, nature des pavements, style des revêtements d'enduits peints et de stucs, typologie du mobilier archéologique contenu sous les niveaux de sol de la Domus aux bucranes et de la Domus à péristyle. A ce stade de l'enquête, la mise en chantier de la Domus aux bucranes doit être envisagée vers 80-60 av. J.-C. La date de sa destruction est certainement liée à celle de la construction de la Domus à péristyle et au rehaussement important du niveau de sol de l'ensemble de la parcelle urbaine de la Schola du Trajan. Cet événement s'est sans doute produit dans les dernières années du $\mathrm{I}^{\mathrm{er}}$ siècle av. J.-C. Le catalogue céramique, particulièrement riche et abondant, est en cours d'élaboration. Il livrera certainement une série d'informations capitales à l'argumentation de ce dossier'.

La Domus aux bucranes représente un ensemble tardo-républicain du plus haut intérêt pour la connaissance d'Ostie, mais aussi pour l'étude de la décoration pariétale dans le Latium, où, hormis le Palatin, il existe peu de vestiges pour cette période. L'emplacement de l'édifice dans le tissu urbain de la colonie romaine, le développement du plan, ainsi que la qualité d'exécution des élévations et des pavements, témoignent de l'élévation sociale de son propriétaire. Les moyens mis en œuvre pour réaliser les revêtements pariétaux de la Domus aux bucranes, sur lesquels la part de rouge cinabre est exceptionnellement élevée, ne devaient en effet pas être à la portée de n'importe quelle famille. S'il n'est à ce jour pas encore possible de déterminer la fonction de la parcelle avant la construction de la domus tardo-républicaine, éventuellement agricole, il est important de constater que l'orientation du plan de la Domus aux bucranes correspond à l'orientation des édifices successifs : la Domus à péristyle et la

9. Les dernières campagnes de documentation ont notamment permis de contester une partie des observations préliminaires publiée par Th. Morard et D. Wavelet, « Prolégomèncs ", loc. cit. (n. 1), p. 777. Lcs fragmcnts de céramique appartenant à la première muilié du I $^{\mathrm{cr}}$ siècle après J.-C., alors incriminés dans la datation tibérienne du chantier de la Domus à péristyle, appartiennent en réalité à une unité stratigraphique contaminée lors d'une intervention postérieure ; ces fragments doivent de ce fait être écartés du corpus de base essentiellement composé de mobilier tardo-républicain. 
Schola du Trajan. De ce fait, les têtes de mur des édifices les plus anciens ont souvent été réutilisées dans les fondations des bâtiments les plus récents. Il est aussi intéressant de constater la superposition de l'hortus de la Domus aux bucranes et celui de la Domus à péristyle.

Les ruines de la Domus aux bucranes témoignent de l'expansion occidentale du tissu urbain de la colonie d'Ostie au cours du $\mathrm{I}^{\mathrm{er}}$ siècle avant J.-C. Cette expansion se fait bien évidemment audelà des antiques limites du castrum primitif, de part et d'autre d'un axe routier important reliant le centre urbain au littoral. Les sondages menés depuis 1938 le long de ce tronçon particulier du Decumanus Maximus ont mis au jour les ruines de plusieurs riches demeures républicaines ${ }^{10}$. Les relations chronologiques entretenues entre ces différents édifices et la fortification tardorépublicaine d'Ostie ne sont en vérité pas bien établies. Ce dossier, de première importance, a été remis récemment à l'ordre du jour par F. Zevi ${ }^{11}$, qui propose de dater du consulat de Cicéron la mise en chantier de la nouvelle enceinte d'Ostie ${ }^{12}$. La relecture de l'inscription qui figurait sur l'attique de la Porta Romana suppose également que cette nouvelle fortification ne fut achevée qu'en 58 avant J.-C., sous le tribunat de P. Clodius Pulcher. Il ne fait cependant aucun doute que le quartier de la Porta Marina était déjà partiellement occupé auparavant.

\section{Perspectives de travail}

Au cours des prochaines saisons, la mission archéologique de l'Université de Lyon II poursuivra l'étude systématique de la parcelle urbaine de la Schola du Trajan. Le projet de recherche envisage non seulement l'analyse de l'ensemble des élévations et des structures mises au jour durant l'hiver 1938-1939, mais aussi la mise en chantier d'une série de sondages indispensables à la compréhension du plan et de l'aménagement des différents édifices concernés ${ }^{13}$. L'organisation de chaque campagne sera bien évi-

10. Voir à ce propos S. Arena Taddei, Ostia Repubblicana. Itinerari Ostiensi I, Rome, 1977.

11. F. Zevi, «Ostie sous la République » dans Ostia, Port et Porte de la Rome antique, Genève 2001, p. 10-19.

12. Sans véritable argument, si ce n'est la technique de construction en opus incertum, la tradition attribuait cette fortification à l'époque de Sylla.

13. L'étude spécifique des élévations de la Schola du Trajan a été commencée, puis abandonnée, par une équipe d'archéologues de l'École française de Rome : E. Bukowiecki, 
demment liée à l'évolution des différents dossiers et discutée au fur et à mesure de l'avancement des investigations sur le terrain. Par principe, et en accord avec la Surintendance d'Ostie, la priorité sera toujours donnée aux ateliers de documentation et de restauration chargés de traiter le mobilier archéologique issu du chantier de fouilles.

En ce qui concerne la Domus aux bucranes, objet de notre discours, plusieurs objectifs ont été définis. A moyen terme, il est ainsi prévu de recomposer l'ensemble du plan et de l'élévation de cet ensemble domestique tardo-républicain, ainsi que de reconstituer, physiquement et virtuellement, une grande partie de son appareil décoratif. Nous attendons également avec impatience les résultats des premières analyses chimiques des pigments et des mortiers utilisés lors du chantier de la Domus aux bucranes. Si quelques sondages périphériques sont encore envisagés dans la zone du tablinum, du péristyle et de l'hortus, l'attention sera dorénavant portée dans la partie la plus septentrionale du bâtiment, vers la zone supposée de l'atrium de la Domus aux bucranes ${ }^{14}$. Par l'étude systématique du mobilier contenu sous les niveaux de pavement des différents édifices, il sera également possible de préciser et d'étayer la chronologie de la mise en chantier et de la destruction de la Domus aux bucranes et de la Domus à péristyle. Des bases de données contenant les ensembles de mobiliers d'époque républicaine et des premiers temps de l'Empire ont été conçues sur un support informatique ; elles représentent une source de renseignements indispensable à la connaissance des phases les plus anciennes d'Ostie, celles qui ont précédé les grands chantiers liés à la construction des ports maritimes de Claude et de Trajan.

La mise en commun des informations recueillies sur les quelques chantiers archéologiques engagés ces dernières années dans le quartier de la Porta Marina permettra certainement de mieux saisir la structure et la densité de l'expansion urbaine,

H. Dessales, J. Dubouloz, « Ostie : relevé et analyse des architectures de la Schola du Trajan (Reg. IV, Is. V, 15-17)", MEFRA 114.1 (2002), p. 454-456; E. Bukowiecki, H. Dessales, J. Dubouloz, « Ostie : l'avant-corps de la Schola du Trajan (Reg. IV, Is. V, 15-17). Stage doctoral d'initiation à l'archéologie du bâti », MEFRA 115.1 (2003), p. 521-530.

14. La distance qui sépare le tablinum et le Decumanus Maximus permet d'envisager un atrium de grande dimension, dont on ne connaît, à ce jour, aucun élément. 
durant l'époque républicaine, le long de ce tronçon du Decumanus Maximus reliant le centre de la colonie au littoral méditerranéen. Il sera alors non seulement temps de définir la fonction de la parcelle urbaine de la Schola du Trajan avant la construction de la Domus aux bucranes, mais aussi de comprendre si la mise en chantier de cette somptueuse demeure privée est antérieure ou postérieure à celle de la seconde fortification républicaine d'Ostie. Doit-on en effet considérer la Domus aux bucranes comme une véritable villa suburbaine, intégrée dans un second temps seulement au tissu urbain de la colonie désormais fortifiée, ou doit-on au contraire envisager que les deux chantiers ont été engagés lors d'une seule et même phase de construction, qui aurait alors vraisemblablement impliqué une réorganisation urbanistique de l'ensemble du quartier de la Porta Marina ? Des éléments de réponse apparaîtront certainement lors de la confrontation de la chronologie revue et corrigée de l'enceinte susmentionnée, désormais reliée à l'époque de Cicéron, avec celle de la Domus aux bucranes et de son appareil décoratif, attachée dans l'état actuel de nos recherches au deuxième quart du $\mathrm{I}^{\text {er }}$ siècle av. J.-C.

Rappelons que le chantier de fouilles, ainsi que les ateliers de documentation et de restauration, sont encore en pleine activité. Il ne fait aucun doute que les prochaines saisons apporteront des éléments décisifs aux différents dossiers de notre étude. Selon le programme naguère établi, une synthèse de nos travaux sera exhaustivement présentée au printemps 2007, lors d'un colloque international organisé, au sein même de l'Université de Lyon II, par les professeurs Fausto Zevi et Jean-Marc Moret, ainsi que par la Surintendance archéologique d'Ostie. Il portera sur les maisons dites de deuxième style, mais qui seront étudiées globalement, c'est-à-dire dans leur structure urbanistique, architecturale et décorative et dans la perspective de leurs fonctions, de leurs commanditaires et des éventuels ateliers itinérants. A cette occasion, il est notamment prévu de présenter une reconstitution de la Domus aux bucranes. Ce double événement, et les réactions qu'il suscitera inévitablement, serviront de fondations à la publication définitive de notre entreprise. Dans cette optique, consciente de la valeur singulière du projet de recherche du site de la Schola du Trajan, la Surintendance d'Ostie nous a récemment réitéré toute sa confiance ; elle a renforcé les liens profes- 
sionnels et amicaux qui unissent nos deux groupes de travail. Qu'elle en soit ici chaleureusement remerciée !

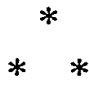

MM. Robert TuRCAN, Henri Lavagne, correspondant de l'Académie, Jean-Paul Morel, correspondant de l'Académie, Robert ÉTIENNE, Jean-Marie DENTZER et Gilbert DAGRON interviennent après cette communication.

\section{LIVRES OFFERTS}

M. Philippe CONTAMine a la parole pour deux hommages :

« J'ai l'honneur de déposer sur le bureau de l'Académie, de la part de son auteur, $\mathbf{M}^{\text {me }}$ Anne Lemonde, maître de conférences à l'Université Pierre Mendès-France (Grenoble II), son livre intitulé Le Temps des libertés en Dauphiné. L'intégration d'une principauté à la Couronne de France (13491408), Grenoble, Presses universitaires de Grenoble, 2002, 437 p. Ce livre offre l'essentiel d'une thèse de doctorat préparée sous la direction de $M^{\text {me }}$ Pierrette Paravy.

L'ouvrage est fort bien documenté, écrit avec fermeté, il n'a rien de touffu ni de confus. L'idée est qu'en "transportant" le Dauphiné en 1349 au futur Charles V, alors même que son grand-père Philippe de Valois était toujours en vie, le dernier dauphin "indépendant", Humbert II, lui léguait une principauté assez démunie et surtout largement dépourvue de cohérence institutionnelle, voire d'unité.

Avec un incontestable sens politique, les dirigeants français de la seconde moitié du $\mathrm{XIV}^{\mathrm{e}}$ siècle donnèrent des structures à cet héritage inorganisé en ménageant autant que possible les susceptibilités régionales : création de l'office de trésorier en 1355, de l'office de gouverneur en 1360, de la Chambre des comptes en 1367, promotion du conseil dauphinois autour des années 1380, émergence des états du Dauphiné à partir de 1388 , ce qui permit, en l'occurrence, de donner la parole aux forces politiques dauphinoises et notamment aux nobles, qui, du même coup, eurent l'impression d'être écoutés et se rallièrent sans état d'âme, au mépris de leurs traditions d'indépendance, à la solution française.

Tout cela fut l'œuvre de serviteurs des rois de France capables et éclairés, que l'on peut en gros rattacher à l'équipe et à l'esprit des fameux Marmousets, fidèles, durant comme après son règne, à la pensée de Charles V. "Les 